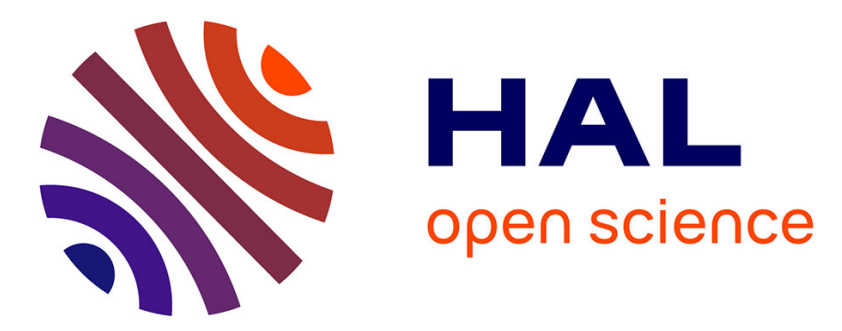

\title{
A new approach to detect early or hidden fungal development in indoor environments
}

Rukshala Anton, Stéphane Moularat, Enric Robine

\section{To cite this version:}

Rukshala Anton, Stéphane Moularat, Enric Robine. hidden fungal development in indoor environments. 10.1016/j.chemosphere.2015.06.072 . hal-02171862

A new approach to detect early or Chemosphere, 2016, 143, pp.41-49.

\section{HAL Id: hal-02171862}

https://hal-cstb.archives-ouvertes.fr/hal-02171862

Submitted on 3 Jul 2019

HAL is a multi-disciplinary open access archive for the deposit and dissemination of scientific research documents, whether they are published or not. The documents may come from teaching and research institutions in France or abroad, or from public or private research centers.
L'archive ouverte pluridisciplinaire HAL, est destinée au dépôt et à la diffusion de documents scientifiques de niveau recherche, publiés ou non, émanant des établissements d'enseignement et de recherche français ou étrangers, des laboratoires publics ou privés. 


\title{
A new approach to detect early or hidden fungal development in indoor environments
}

\author{
Rukshala Anton $^{1 *}$, Stéphane Moularat ${ }^{1}$ and Enric Robine ${ }^{1}$
}

${ }^{1}$ Centre Scientifique et Technique du Bâtiment(CSTB), 84, Avenue Jean Jaurès Champssur-Marne 77447 Marne-la-Vallée Cedex 2

*corresponding author: rukshala.anton@ @stb.fr

\section{Introduction}

Among the pollutants from indoor environments figure micromycetes or mold. This microbial contamination is not without consequence. Indeed, fungi are growing to the detriment of the colonized support, ultimately with possible degradation of its mechanical properties in particular.

In addition to the biodegradation of the products which they colonize, mold can induce occupants various diseases, in particular respiratory pathologies such as allergies, infections or toxi-infections(Kuhn, Hana et al. 1995). Thus, several epidemiological studies have shown an association between the increasing prevalence of asthma or respiratory symptoms on one hand, and the presence of molds (or excessive humidity) in the internal spaces on the other hand(Garrett, Rayment et al. 1998; Kilpelainen, Terho et al. 2001; Moularat, Hulin et al. 2011; Hulin, Moularat et al. 2013).

Studies in Europe and North America on the fungal contamination of dwellings, showed that $14-35 \%$ of the investigated environments were concerned (Tsongas 1994; Pirhonen, Nevalainen et al. 1996; Escamilla-Garcia 1997). This finding was corroborated by the Observatory of Indoor Air Quality in 2008. Thus, 15\% of French real estate present fungal infections, among these $15 \%$, there are $2 \%$ of cases (610,000 units) with contaminated surfaces over $1 \mathrm{~m}^{2}$ (Moularat, Robine et al. 2008).

Thus, provide tools for monitoring and / or quick diagnosis of a fungal development constitutes a major issue which makes it even more urgent that requirements to reduce 
energy consumption in buildings expressed by the government during the Grenelle 2 will lead to buildings whose permeability has been reduced and therefore more susceptible to dysfunction of aeration devices. However, if improving the tightness of the building envelope is effective, it is feared that excess moisture whether it is of accidental origin, related to the activities of the occupants, or a dysfunction of the ventilation; promote conditions convenient to fungal growth.

In an optics of early detection, the work of CSTB rely on the emission, in the early hours of fungal development, of Microbial Volatile Organic Compounds (MVOCs). These diffuse into the environment and constitute a specific biochemical fingerprint whose measure is exploitable for diagnosis or monitoring of confined spaces.

Techniques conventionally used to diagnose a fungal contamination in an environment are based on visual examination and culture of fungal spores in the air. While in many situations of infestation, this approach is efficient, it does not allow for much the detection of contamination "hidden" (behind a partition on ventilation filters for example) or early (in the early stages of mold growth).

However, early in their development, fungi emit volatile compounds (MVOCs) from either their metabolism or degradation of the material by enzymes or acids they produce (Wessen, Ström et al. 1995; Korpi, Pasanen et al. 1997; Korpi, Kasanen et al. 1999; Wilkins 2002; Moularat, Robine et al. 2008; Moularat, Robine et al. 2008).

Moreover, unlike spores, these gaseous compounds diffuse into the environment without being restrained by the media.

Thus a method, based on the detection of several VOCs from fungal metabolism, has been proposed as a Fungal Contamination Index (FCI). The presence and / or absence of different tracers are taken into account, the index is incremented according to their specificity towards species /substrate (Moularat 2007). The FCI can finally decide on an active fungal growth, including cases of early or "hidden" contaminations.

This tool has already been used in many studies:

- Applied to gaseous samples taken at the National Housing Campaign Observatory Air Quality (OQAI) it has a description of the state of fungal contamination of French housing. 
- Used in an epidemiological study, the association between fungal growth and asthma or symptoms similar to chronic bronchitis(Hulin, Moularat et al. 2013) has been demonstrated

- Applied to 94 homes in the area of Clermont- Ferrand in the context of another epidemiological study (ISAAC), this tool has also demonstrated the relationship between fungal development and childhood asthma(Hulin, Caillaud et al. 2010).

Studies in collaboration with agencies responsible for safeguarding the heritage such as the Research Laboratory of Historical Monuments, National Archives or the National Library of France, have also shown interest in biochemical fingerprints as a means of detecting fungal(Joblin, Moularat et al. 2010; Nguyen, Moularat et al. 2012; Hulin, Moularat et al. 2013).

Preliminary tests performed using different strains covered by the study of Joblin et al 2010 made it possible to obtain specific responses using polymer sensors exposed to mouldy environments. Thus in the laboratory, the sensors studied were thus able to distinguish contaminated from non-contaminated environments.

However, the use of a profile forces to compare it with a reference (control) what is not applicable in indoor environments. Besides, if these Electronic Conductive Polymers (ECP) layers showed themselves effective for the detection by the identification of a global VOC fingerprint emitted by mould, they are not still enough selective to realize the identification of every COV, essential for the index calculation. So, these sensors remain insufficiently specific to permit their use in situ.

Regarding these stakes, having a tool which can be installed in buildings and capable in supplying an almost immediate information about a possible fungal development could be a major step forward.

Now, if the FCI was widely used, the analysis of VOCs' by Gas Chromatography coupled with Mass Spectrometry (GC-MS) necessary for its calculation, is incompatible with a strategy of real-time control of the indoor environments.

In this context, we propose a monitoring beacon. Our efforts of research concerned the miniaturization of the analytical chain for portable and reliable applications at moderate costs. The final system consisted of two modules of pre-treatment of samples (a module of concentration also enabling air collection and a module of separation) and of a VOCs' 
analysis module. Laboratory experiments have been performed to validate that each of these modules has the desired features to collect, separate and detect VOC from the Fungal Contamination Index so that the microsystem could calculate it.

\section{Materials and Methods}

\subsection{Description of modules constituting the microsystem}

\subsubsection{Concentration module}

The concentration microstructure developed in this study consisted of a silicon substrate in which $60 \mathrm{~mm}$ length microchannels with a $500 \mu \mathrm{m}$ width were etched. The sealing with a glass substrate enabled the realization of closed cavities. The microstructure was then packed with a granular adsorbent, Tenax TA, with an average diameter of $120 \mu \mathrm{m}$. Fluidic ports adapted to this type of microstructure enabled to equip the micro-module openings with capillaries for pump connection and air circulation through the structure. Tenax TA tube that is classically used for FCI calculation contains a $1 \mathrm{~cm}^{3}$ volume with average bead diameter of $300 \mu \mathrm{m}$. The beads' size used for the microstructure (average diameter of $120 \mu \mathrm{m}$ ) enabled to increase the specific surface. Consequently, in spite of the volume decrease $\left(0.20 \mathrm{~cm}^{3}\right)$, the equivalent active surface is preserved. So, the concentration efficiency (retention and drilling volume) of this microstructure remains equivalent to a classic tube.

\subsubsection{Separation module}

The micro-column developed also consisted of a silicon substrate in which a microchannel is etched. The sealing with a glass substrate enabled the realization of this microchannel. The fabricated micro-chip with a $5 \mathrm{~m}$-long, $150 \mu \mathrm{m}$-wide, $200 \mu \mathrm{m}$-deep separation column was coated with polydimethylsiloxane (Sylgard® 184, Dow corning) as the stationary phase using static coating method. Indeed, because compounds to be detected in the FCI belong essentially to alkenes, ketones and esters and are polar, an apolar stationary phase was chosen. As for the concentration microstructure, adapted fluidic connectors enabled to equip the micro-module's openings with capillaries. 


\subsubsection{Detection module}

Interdigitated Electrodes (IDE) with different geometries were implemented in the array to increase the sensor dynamic range variability. Conducting polymers were chosen as sensing materials as they enable differentiation between contaminated and noncontaminated environments (Joblin, Moularat et al. 2010). Presently, 8 different polymers are used.

\section{Polymer deposition substrate}

The IDE are made up by two overlapping metal comb structure. They were obtained by successively cathodic pulverization of a $50 \mathrm{~nm}$ thickness chromium layer and a $1 \mu \mathrm{m}$ thickness golden layer at $24^{\circ} \mathrm{C}$ in an argon plasma. These IDE were made in cleanroom by the ESIEE Engineering. Two geometries of IDE with respectively 6 and 26 bands were used. The 6 and 26 IBE have respectively an electrode width of $260 \mu \mathrm{m}$ and $60 \mu \mathrm{m}$ and a gap size between electrodes of $300 \mu \mathrm{m}$ and $60 \mu \mathrm{m}$.

\section{Deposited polymers onto IDE}

During the experiments, 5 types of electronic conducting polymers (ECP), either in solution, such as PEDOT-PSS (Poly(Ethylenedioxythiophene)-Poly(styrene-sulfonate)) or electrochemically deposited (using different counter-ions or adding functional group to the main polymer ring), were applied to the interdigitated band electrodes.

The PEDOT-PSS (Clevios ${ }^{\mathrm{TM}}$ PH 1000) comes from H.C. Starck. Its concentration is $1.3 \%$ in weight, and the ratio (in weight) of PSS over PEDOT is 2.5 in solution. This polymer in solution was applied by drop-coating to the 6 IDE using the nose cone of a micropipette. In order to vary its structure and morphology, three thermal annealing, respectively $80^{\circ} \mathrm{C}, 100^{\circ} \mathrm{C}$ and $140{ }^{\circ} \mathrm{C}$ during 5 minutes, were studied. The non-annealed (PEDOTPSSNA) and annealed PEDOT-PSS (PEDOTPSS80; PEDOTPSS100 and PEDOTPSS140) were considered thereafter as 4 distinct polymers. 
The four other polymers were applied by electropolymerization to the 26 IDE until percolation was achieved (junction of a polymer between the two electrodes) using an assembly of three electrodes. These polymers have been synthesized from 4 fresh monomers from Sigma Aldrich, France (Pyrrole, N-methylpyrrole, 3-methylpyrrole and methyl-1H-pyrrole-3-carboxylate) at a concentration of $0.05 \mathrm{M}$ in two couples of counterion $(0.1 \mathrm{M})$ /solvent (octane sulfonic acid sodium salt /deionized water for pyrrole and lithium perchlorate/acetonitrile for the 3 other monomers). All reagents were used of analytical grade.

The five polymers are listed in Table 1. 
Table 1 : List of the 5 conducting polymers deposited on sensor substrate

Drop-coated polymer (Commercial solution of PEDOT-PSS)

\begin{tabular}{c|c|c}
\hline Polymer & Counter-ion & $\begin{array}{c}\text { Abbreviation for Principal } \\
\text { Component Analysis (PCA) }\end{array}$ \\
\hline Poly(Ethylenedioxythiophene) & Poly (styrene-sulfonate) & PEDOTPSSNA \\
\cline { 2 - 3 }$($ PEDOT) & PEDOTPSS80 & PEDOTPSS100 \\
\cline { 3 - 3 } & &
\end{tabular}

Electrodeposited polymers

\begin{tabular}{|c|c|c|}
\hline Polymers & Counter-ions & $\begin{array}{l}\text { Abbreviation for Principal } \\
\text { Component Analysis (PCA) }\end{array}$ \\
\hline Polypyrrole & Octane sulfonate & PyOS \\
\hline Poly (3-methylpyrrole) & & 3Methylpyr \\
\hline $\begin{array}{l}\text { Poly (N-methylpyrrole) } \\
(1 /)\end{array}$ & Perchlorate & Nmethylpyr \\
\hline $\begin{array}{c}\text { Poly (Methyl-1H-pyrrole-3- } \\
\text { carboxylate) } \\
\|_{C}{ }_{0}{ }^{\mathrm{CH}_{3}}\end{array}$ & & Methyl1Hpyrrole3carboxylate \\
\hline
\end{tabular}




\subsection{Description of modules' validation setups}

\subsubsection{Biological material}

The selected mould specie for these experiments is a strain from the Institute of Hygiene and Epidemiology in Brussels (IHEM 05077) : Aspergillus niger.

\subsubsection{Chemical materials}

Twenty-one standard compounds (SIGMA-ALDRICH $\rightarrow$ 95\% purity) representing different chemical VOC families and that are characteristics or non characteristic of fungal metabolism, were identified. Are studied a linear alkane (VOC 1), 5 alkenes (VOC 2, VOC 3, VOC 4, VOC 5 and VOC 6), 5 linear primary alcohol (VOC 7, VOC 8, VOC 9, VOC 10 and VOC 11), 2 branched primary alcohols (VOC 12 and VOC 13), 2 secondary alcohols (VOC 14 and VOC 15), 3 ketones (VOC 16, VOC 17 and VOC 18) and rings (VOC 19, VOC 20 and VOC 21). Each of these 21 molecules has been tested separately.

\subsubsection{Emission chambers}

Modules validation tests were performed on $300 \mathrm{ml}$ emission chambers. These chambers are used to generate experimental atmospheres and are made of materials that remain inert in presence of VOC (glass and PTFE).

Chambers contained either contaminated substrates (glass fabric) by mold for preconcentration and separation modules validation or chemical compound for polymer sensors validation.

For the mold contaminated chamber, glass fabric was used as growth substrate. This material was cut and sterilized $\left(121^{\circ} \mathrm{C}, 45 \mathrm{~min}\right.$, wet heat) before adding distilled water. A pool of $5 \mathrm{~mL}$ of water kept the chamber water saturation during the test (RH>99\%). The moisture level was maintained at saturation throughout tests. The obtained load rate was $7.10^{-2} \mathrm{~cm}^{2} / \mathrm{cm}^{3}$. The spore suspension preparation used for surface contamination 
by Aspergillus Niger was carried out by pouring $50 \mathrm{~mL}$ of ultrapure water on the strain subcultured on oats. Air filtered through activated charcoal is passed for 30 minutes in each chamber. Then each contaminated chamber was placed in a stove for 7 days at $25^{\circ} \mathrm{C}$ in total darkness.

For polymer sensor tests, two types of atmospheres were created, one named reference in order to generate filtered air and the second one with a VOC. To constitute a reference chamber, Tenax TA tubes were introduced at each end of valves, then they were closed. Activated carbon was introduced to filter the incoming air. To realize a VOC emission chamber, Tenax TA tubes were introduced at each end of valves, then they were closed. $300 \mu \mathrm{L}$ of each VOC were introduced there to create a saturated atmosphere. Then the chamber was placed in a stove at $25^{\circ} \mathrm{C}$ for at least 30 minutes prior to a test.

\subsubsection{Analytical system}

Pre-concentrator and micro-column were validated using the GC-MS (6890 Gas Chromatography (GC) equipped with a 5973 Mass Spectrometry (MS) detector, Hewlett Packard, USA). A sample of $5 \mu \mathrm{L}$ is injected. The GC column was a HP-SMSUI fusedsilica capillary column (60 m x $0.25 \mathrm{~mm}$ i.d. x $0.25 \mu \mathrm{m})$ with a stationary phase of $5 \%$ Phenyl and $95 \%$ of Methylpolysiloxane. The carrier gas was helium (>99.995\%) at a flow rate of $0.5 \mathrm{~mL} \cdot \mathrm{min}^{-1}$. The column temperature program was an isothermal with a temperature of $40^{\circ} \mathrm{C}$ during $10 \mathrm{~min}$. The "SIM" ("Single Ion Monitoring") mode was used. Once all the corresponding chromatograms and their associated spectra had been analyzed, the results were compared with NIST mass spectrum library(Nist'98 1998).

\subsubsection{Sensors' study setup}

The sensors'study was performed from the setup which is part of the aforementioned microsystem (Moularat, Joblin et al. 2010; Moularat, Joblin et al. 2011).

This bench test is mainly constituted of 2 emission chambers, a chamber in which are placed polymer sensors (called sensor chamber) and a system dedicated to signal processing. 


\section{SENSOR CHAMBER}

The sensor chamber was developed to isolate the sensitive layers of polymer sensors from the surrounding air and to expose them solely to air samples circulating in the microsystem. To reduce background noise, non-VOC emitting materials under the experimental conditions of this study were selected. Thus, the sensor chamber was made of stainless steel and seals were made of Teflon (PTFE). This enabled air circulation over sensors at a 1 mL.min-1 flow rate.

\section{SIGNAL PROCESSING}

Four IDE with polymer were placed in the sensor chamber in order to be used as the detection module in the microsystem. The operating principle of these sensors is based on conductivity change of conducting polymers induced by the adsorption/absorption of gas on its surface. This adsorption/absorption depends on the VOC affinity with the active sites of the polymer layer. To convert this conductivity change in a measurable signal, each sensor was placed in a Wheatstone bridge in order to measure an electric resistance change.

\subsection{Modules' validation protocols}

\subsubsection{Concentration validation module}

The test protocol for the concentration microstructure validation required the use of a sample with fungal contamination (emission chamber containing the glass fabric contaminated with Aspergillus niger performed in triplicate). The test protocol consisted in collecting air from the chamber through the pre-concentrator for $30 \mathrm{~min}$ at a flow rate of $6.2 \mathrm{~mL}$. $\mathrm{min}^{-1}$ by a diaphragm pump SP $725 \mathrm{EC}$. The sample from the pre-concentrator was then desorbed at $140{ }^{\circ} \mathrm{C}$ for $30 \mathrm{~min}$ and extracted via the pump into a chamber containing a sample vial. $5 \mu \mathrm{L}$ of the sample from the pre-concentrator contained in the vial was then injected and analyzed by GC-MS following the protocol detailed above. Three consecutive measurements were realized. 


\subsubsection{Separation validation module}

GC-MS used for the pre-concentrator validation was employed by replacing the conventional chromatographic column by the micro-column. A sample with fungal contamination (emission chamber containing the glass fabric contaminated with Aspergillus Niger) has also been used in the test protocol of the micro-column. As for the concentration module validation, $5 \mu \mathrm{L}$ of the sample contained in the vial was then injected and analyzed by GC-MS following the same protocol. Three consecutive measurements were realized.

\subsubsection{Detection module validation}

The study of the sensors' response was alternately performed with two emission chambers, one reference with activated charcoal ( 2 min exposure) and the other one with VOC (15 sec exposure). The air used between injections was filtered on activated charcoal and a Tenax tube. This filtered air enabled quick desorption of VOCs that have penetrated into the polymer in order to find a stable baseline.

Resistance responses of each triplicate sensor to each vapour exposure were recorded three times and the percentage change against the baseline were calculated using:

$$
\% \frac{d R}{R}=\frac{\left(R_{\text {sensor exposed to VOC }}-R_{\text {sensor non exposed to VOC }}\right)}{R_{\text {sensor non exposed to VOC }}} \times 100
$$

A signal was considered as a response of the sensor to the stimulus when its intensity exceeded the detection limit defined by $\mathrm{DL}=$ mean (background) $+3 \mathrm{x}$ standard deviation (background).

\subsubsection{Interpretation of sensor data (Principal Component Analysis)}

To perform Principal Component Analysis (PCA), the software of data analysis named SPAD version 3.5 (Decisia) was used. PCA is a technique of statistical description leading to graphical representations of a data table contents: simultaneous description of links between variables (VOCs) and similarities between individuals (ECP) (projection in a small space). This representation enables therefore to highlight the existence of variable groups or individuals, in our case the distinction between VOCs. The PCA resulted in a 
reduction of information by grouping the variables into independent factors: the principal components that are linear combinations of variables. The PCA is described in detail in the book Statistique exploratoire multidimensionnelle (Lebart, Morineau et al. 1997).This technique enables through statistical calculations to determine the most representative plan in terms of our data without making an a priori on results.

\section{Results}

\subsection{Concentration module validation}

In order to validate the pre-concentrator, the air from the chamber contaminated by Apergillus niger was sampled through the module. It was then heated at $140{ }^{\circ} \mathrm{C}$ to desorb the sampled VOCs in a chamber with a vial. The sample from the pre-concentrator contained in the vial was then analyzed on GC-MS. Eight compounds identified as coming from the fungal metabolism(Moularat, Robine et al. 2008) were then searched. After chromatogram analysis, all these VOC were found (Fig. 1).

\section{VOC 21}

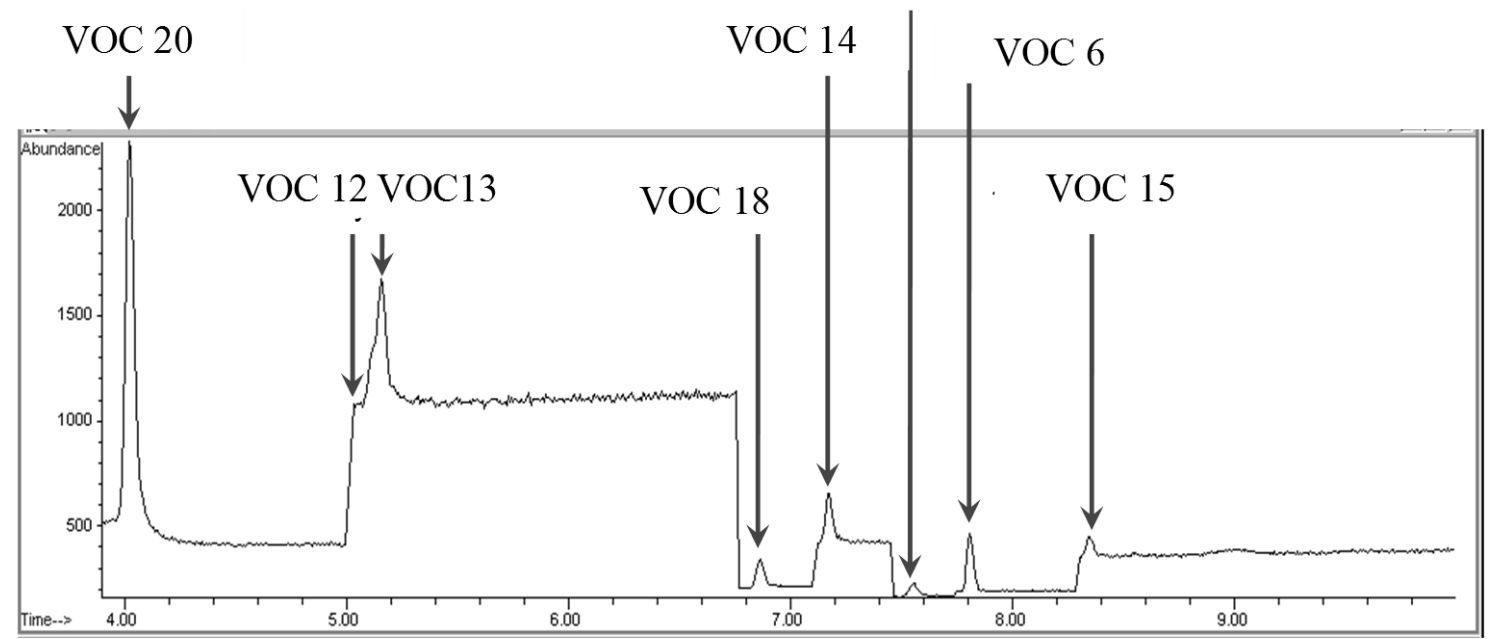

Fig. 1 : CHROMATOGRAM OF EMISSIONS FROM A CHAMBER CONTAINING ASPERGILLUS NIGER CONTAMINATED GLASS FABRIC (CHAÎNE HEWLETT PACKARD MODE SIM)

The results repeatability was checked with three successive measurements. Thus, the results enabled to validate both the trapping and desorption of the compounds at $140{ }^{\circ} \mathrm{C}$. 


\subsection{Separation module validation}

Once the chromatographic micro-column was realized and filled with PDMS, tests were carried out to verify the retention and separation effectiveness of various targeted compounds of the study. In order to validate this module, the air from the chamber contaminated by Apergillus niger collected in the vial is analyzed by GC-MS. After chromatogram analysis, all VOCs' were found (Fig. 2)

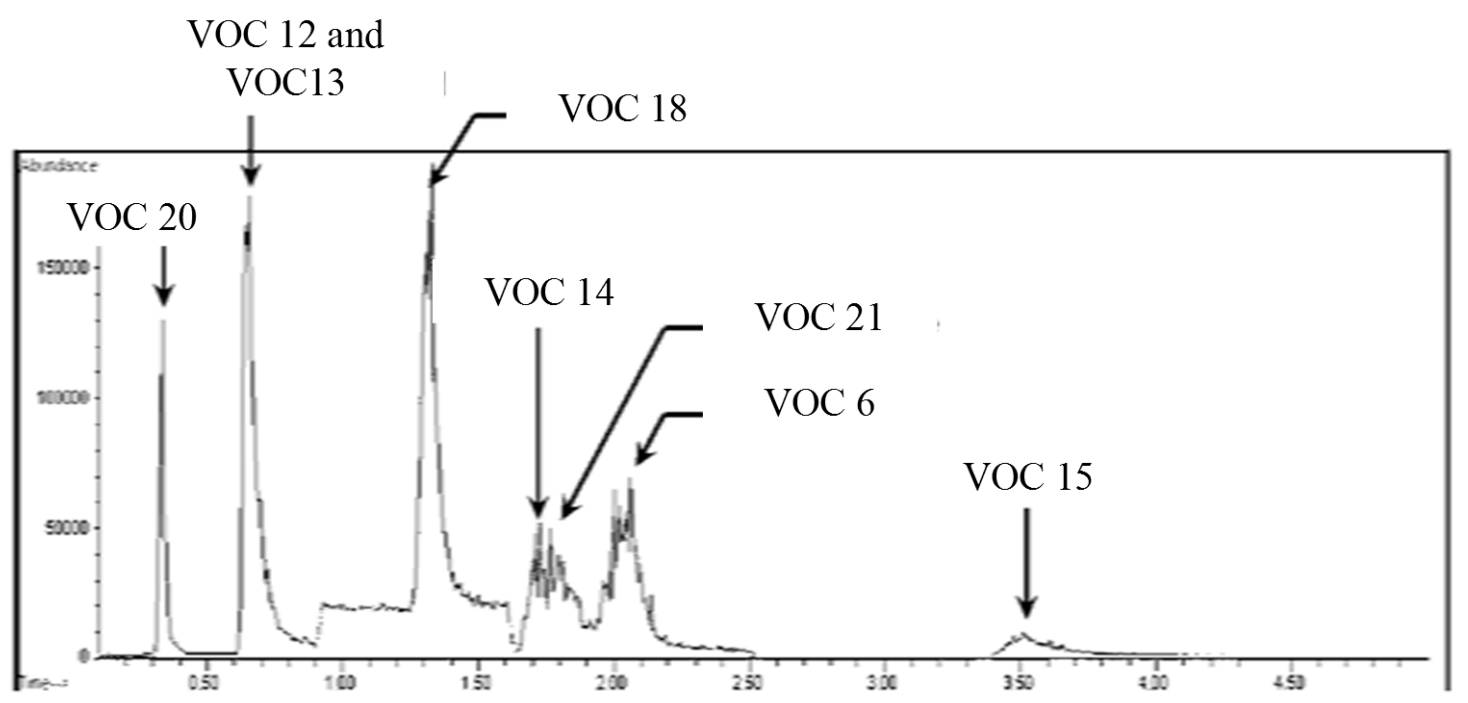

Fig. 2: Chromatogram of EMISSIONS fROM A Chamber CONTAINING ASPERGILlUS NIGERCONTAMINATED GLASS FABRIC (CHAîNE HEWLETT PACKARD - MODE SIM)

Thus, different retention times were found for the various families, proving the efficiency of the micro-column separation. These tests proved that the micro-column was able to separate eleven targeted VOC for fungal contamination index calculation. The separation tests in this study confirmed that this stationary phase provided sufficient separation for our problem, despite the short length of the micro-column.

\subsection{Sensor array responses}

All the 8 sensors responses were the subject of a statistical study. The results are in the form of a matrix of 21 rows or individuals / comments (VOCs) and 8 columns or variables (ECP).The intersection of a row and a column corresponds to average resistance response $(\mathrm{dR} / \mathrm{R}(\%))$ for an ECP and a given VOC.

The dimensions of this data matrix were such that it was impossible to detect directly any similarities in statistical behavior between VOC (individuals) or ECP 
(variables).Principal Component Analysis (PCA) was thus chosen to analyze these results. PCA is a statistical description technique leading to simplified graphical representation of the contents of a data table in order to highlight the existence of variable groups or individuals, in our case the discrimination between fungal and non-fungal VOCs.

The PCA leads to the information reduction by grouping independent factors in variables called principal components. They are defined by linear combinations of variables representing the maximum variance in the scatterplot (all the values). There are as many factors as variables. Two factors define a plane on which the points (individuals: COV) or vectors (variables: ECP) are projected, enabling the construction of two spaces, a vector space of the variables and a space of individuals. This technique enables optimum and similar graphical representations of the scatterplot representing the data matrix without any preconception and without any bias. The proximity of two vectors (variables) indicates a strong linear correlation between these two variables, as does the proximity of two points. Also the proximity between two points indicates a strong correlation between two individuals. Groups of individuals and defined variables thus give a new arrangement of the data structure which was not expressed at first sight. The PCA is normalized (centered on reduced data).

The PCA has been used as an exploratory tool to visualize and explain the relationships between data.

Initially the PCA enables, through statistical calculations, to determine the most representative coordinate of our data. Factor 1 was representative of more than 3 variables out of 8 . The first two selected factors accounted for $62.01 \%$ of the global variance, the principal plane thus grouping most of the information.

In order to identify the variables responsible of the observed behavior, 8 variables (ECP) were projected on the correlation circle in the main plane (Fig. 3). The length of the vectors obtained informed on their representation in the circle. Thus, a variable was better represented in the plane if the vector norm was close to the unit circle with a value of 1. 


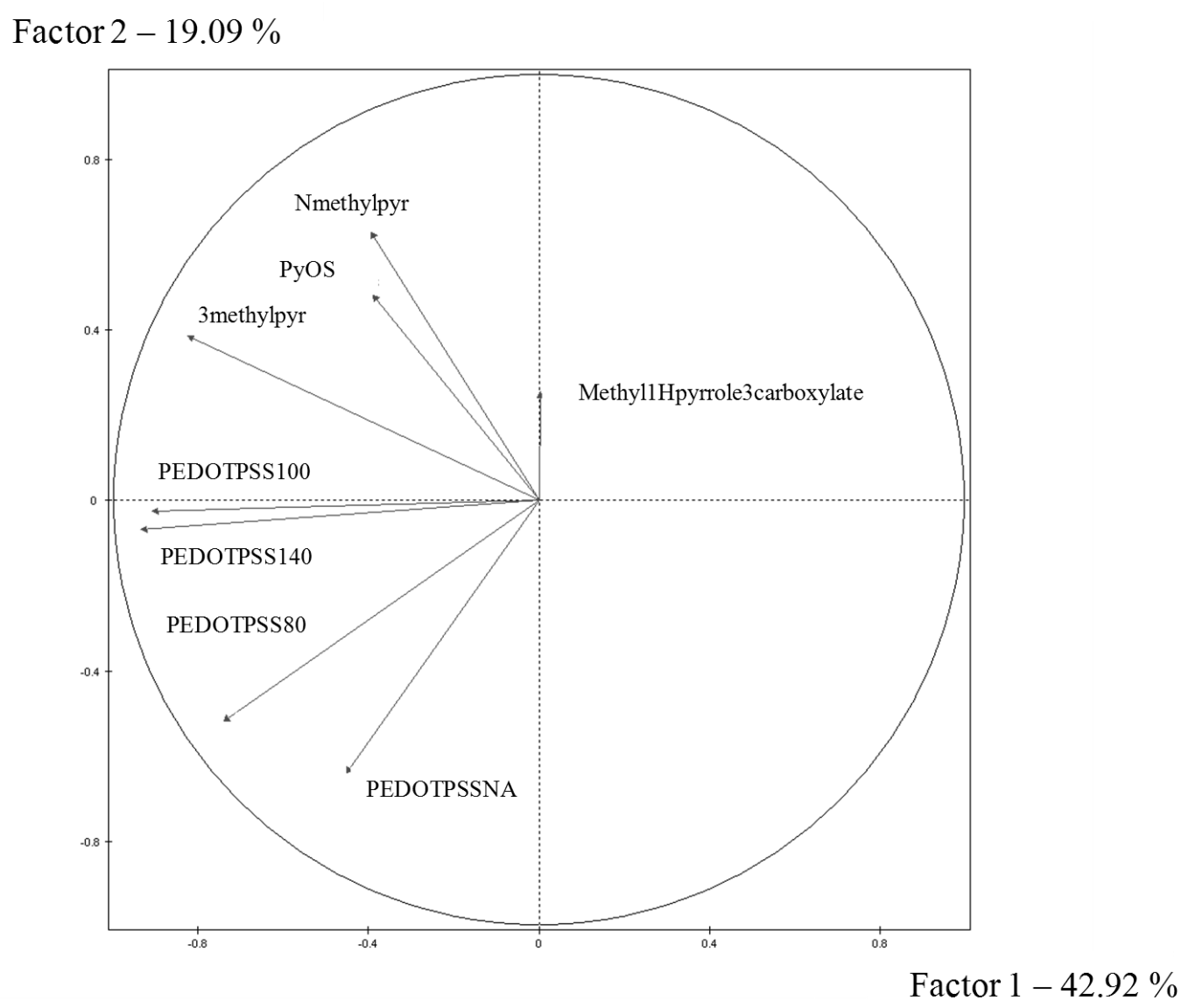

Fig. 3: 8 ECP WERE PROJECTED ONTO A CORRELATION (1 ${ }^{\text {ST }}$ AND $2^{\text {ND }}$ FACTORS)

The second axis opposed drop coated ECP (PEDOTPSS Non Annealed and those annealed at $80{ }^{\circ} \mathrm{C}, 100{ }^{\circ} \mathrm{C}$ and $140{ }^{\circ} \mathrm{C}$ ) to those deposited electrochemically (polypyrrole and its derivatives).

PEDOTPSS NA and PEDOTPSS annealed at $80{ }^{\circ} \mathrm{C}$ and $140{ }^{\circ} \mathrm{C}$ were not correlated and had different sensitivities. However, the PEDOTPSS annealed at $100{ }^{\circ} \mathrm{C}$ and the one annealed at $140{ }^{\circ} \mathrm{C}$ had a correlation, that meant they presented statistics similarities. This showed that both ECP gave similar information. Indeed, for VOCs for which both ECP reacted, response profiles were identical.

PEDOTPSS annealed at $140{ }^{\circ} \mathrm{C}$ had greater sensitivity for most of the tested VOCs that the one annealed at $100{ }^{\circ} \mathrm{C}$, this is due to the residual water content.

Concerning polypyrrole and its derivatives, poly (N-methylpyrrole) and unsubstituted polypyrrole were correlated. Both ECP gave similar information on sensors. 
There is independence between the poly (methyl-1H-pyrrole-3-carboxylate) and the PEDOTPSS100 (perpendicular vectors), and between the poly (N-methylpyrrole) and the PEDOTPSSNA, showing that ECP provided additional information.

The main plane was presented in Fig. 4, the points represented different VOCs. The size of the dots is proportional to their representation in the plane, which defines the quality of their projection on this plane. Thus the larger the point, the better it was represented. With the exception of hexane and 1-octene, which were less represented in this plane, a discrimination between the fungal metabolism characteristic VOCs' (closed circles) and the other (open circles) was observed.

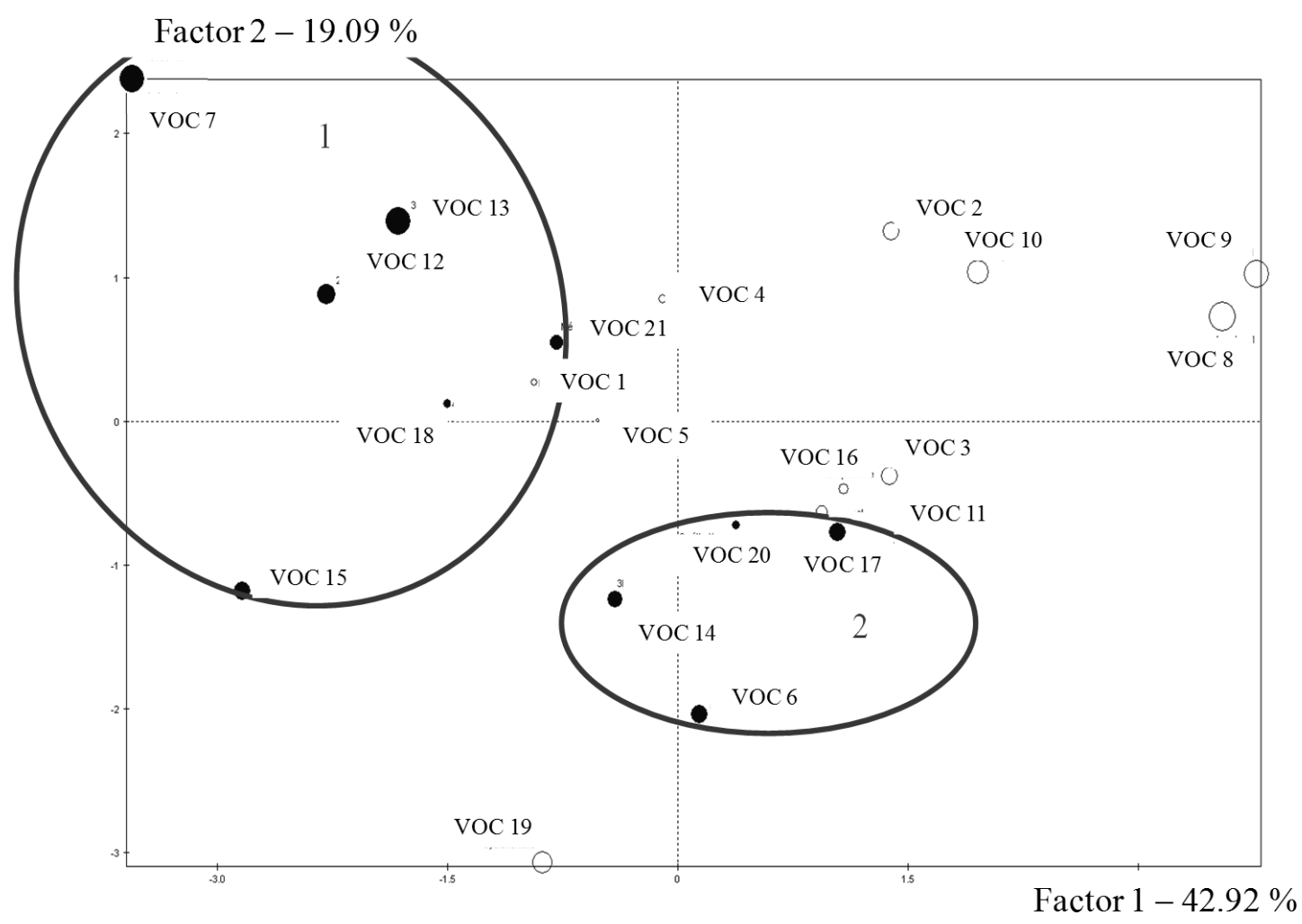

Fig. 4: PCA'S PRINCIPAL PLANE FROM 21 VOCS' ET 8 ECP (DISTINCTION BETWEEN FUNGAL METABOLISM CHARACTERISTIC VOCS' (FILLED CIRCLES) AND THOSE NOT CHARACTERISTIC FROM FUNGAL METABOLISM (EMPTY CIRCLES)) (OBTAINED BY SPAD 3.5)

Thus, separation between fungal metabolism characteristic VOCs and those which are non characteristic had been realized. This discrimination had been verified with a Hierarchical Classification which confirmed that VOC 1 and VOC 5 are excluded from fungal metabolism characteristic VOCs. The results of this classification are represented 
by two ellipsoids (circles in Fig. 4). The measurement of $d R / R$ enabled to discriminate these VOCs.

Moreover, within these two groups (circled), the dots are separated, the tested ECP matrix thus enabled the identification of each tracer in the gas mixture. The two axes were responsible for this separation, showing that all the ECP was the cause of this result.

All the responses of all based on the different sensors were listed and enabled to create a sensor array. The number 1 has been assigned to a negligible response profile, the number 2 to a positive response profile and finally the number 3 to a negative response profile.

These differences in sensitivity and response patterns had contributed to the creation of a specific signature for VOCs. Thus, the responses matrix defined provided 14 specific signatures for 14 VOCs. VOCs (VOC 9 and VOC 10; VOC 2 and VOC 4; VOC 5, VOC 19 and VOC 1) had common signatures.

\section{DISCUSSION}

The objective of this research was to develop a miniature, autonomous enabling the detection of fungal contamination in indoor environments. Three micro-modules were therefore designed for the realization of this microsystem: a pre-concentration module, a separation one and finally a detection one.

Thus, regarding the pre-concentration micro-module, the micro-structure was sized to maintain the characteristics of a conventional sampler. The concentration efficiency (retention and volume of drilling) of this microstructure is still equivalent to a conventional collection tube.

Concerning the separation module, the length of the micro-column $(5 \mathrm{~m})$ is shorter than that of a conventional column $(60 \mathrm{~m})$. Despite this decrease, better retention efficiency can be achieved with thick films of polydimethylsiloxane, enabling the analysis of light compounds such as selected tracers. Separation tests in this study confirmed that this 
stationary phase provides sufficient separation to our problem, despite the short length of the column.

However, it is difficult to compare these results with the literature since there are no studies on fungal development tracers. Nevertheless, other more typically desired compounds, such as benzene and toluene, are also present in samples contaminated with molds. It was thus possible to compare the retention efficiencies for these two molecules with other studies developing PDMS micro-column. Thus, Serrano et al., In 2009, from a $3 \mathrm{~m}$ micro-column, get a 10 s retention difference (Serrano, Reidy et al. 2009). Reidy et al. in 2006, with columns of 0.5 to $3 \mathrm{~m}$, obtained retention times between 15 and $40 \mathrm{~s}$ depending on the length of the column (Reidy, Lambertus et al. 2006). Retention time variations obtained in our tests were the same order of magnitude as that these two compounds were desorbed, in our test conditions, with $24 \mathrm{~s}$ intervals.

Concerning the detection module, preliminary tests from polymer sensors enabled to discriminate laboratory contaminated environments from sterile one (Joblin, Moularat et al. 2010). These findings were reinforced by other studies which discriminate different laboratory contaminated environments (Gibson, Prosser et al. 1997; Canhoto, Pinzari et al. 2004; Dutta, Morgan et al. 2005; Hamilton, Hepher et al. 2006; Bailey, Pisanelli et al. 2008).

However, in these studies, environments' discrimination was based on the detection of a global fingerprint, which does not distinguish the nature of VOCs. The polymer sensors were insufficiently specific to enable the detection of each VOC separately, which is essential for fungal contamination index calculation (Joblin 2011). Thus, this approach was considered unsuitable for in situ sampling. 
Initial work on conductive polymer chains was carried out to optimize their sensitivities and selectivities for various target compounds ( $\mathrm{Li}$, Santhanam et al. 2007; Bailey, Pisanelli et al. 2008). A second work on PEDOT-PSS thermal treatment was then conducted, still in order to optimize the sensitivity and selectivity.

As in the study of Li et al. 2007 with the polythiophene, the study of substituent nature (methyl vs methyl ester) on pyrrole based sensors' response enabled to see that, in these conditions, the sensitivity and selectivity of the compounds may vary. Thus, the addition of a polar substituent has reduced the number of VOCs that interact with the sensitive layer and then allowed to increase the selectivity of pyrrole. The sensitivities were different, depending on the thermal annealing of ECP. Moreover, generally, the sensitivity is increased for PEDOT-PSS annealed at $140{ }^{\circ} \mathrm{C}$. With such treatment, the water contained in the PEDOT-PSS must be further removed, explaining the greater resistance drop compared to those observed in the other treatments at $80{ }^{\circ} \mathrm{C}$ and $100{ }^{\circ} \mathrm{C}$. According Xiong et al., polymer morphology would change during this removal so that the ECP is proving to be more selective than the untreated polymer (Xiong and Liu 2012). Surprisingly, an increased sensitivity of unannealed PEDOT-PSS was observed for VOC 1 and VOC 19 even if it contained more water. Therefore, the sensitivity to both non polar compounds should be lower.

The study of the influence of annealing treatment on sensors' response showed that the sensitivity and selectivity of the compounds vary. Thus, the heat treatment has reduced the number of VOCs that interact with the sensitive layer and then increased the selectivity of the polymer. As for the addition of a substituent, these differences in sensitivity and selectivity contribute to the creation of a specific signature for VOCs.

Thus, the tested sensor array enabled to yield specific signatures for 14 out of the 21 VOCs tested. For 7 other VOCs with identical signatures, the use upstream the separation 
module allowed to discriminate them. Thus, the coupling of the retention times with the chemical signature enabled identification of the 21 VOCs tested.

So, the developed modules are suitable for an analytical micro-system that integrate air sampling, transfer, separation and analysis steps.

The modules tested in this study enabled to consider early detection of fungal growth in an indoor environment. This beacon coupled with the benefits of the fungal contamination index (detection of recent contamination and/or hidden, specificity of detection) makes it a useful tool for monitoring the microbiological quality of enclosed spaces. In addition, the modularity of the system, including the ability to vary both the elements of polymers detection and retention time of interest, and therefore the nature of VOC to analyze, allows expanding its use to other pollutants.

\section{CONCLUSION}

The aim of this study was to find a new approach to detect early or hidden fungal development in indoor environments adapted to real-time monitoring. For this purpose, three micro-modules were developed and validated in order to integrate them in miniaturized monitoring device.

Thus, the first developed module, namely the one for air sampling, allowed the trapping of 8 tracers and simultaneous desorption of compounds. The second-one enabled the separation of 7 tracers among the 8 tested VOC: only 2 isomers are coeluted. The last module, the one of detection using 8 polymer sensors array, enabled to obtain 14 specific signatures for 14 VOCs. Some VOCs have common signatures. However, the use of the micro-column in the microsystem enables the separation of these molecules. Coupling of retention times with chemical signature enables the identification of 21 VOCs.

These modules have thus shown relevance towards chemical targeted compounds with retention, separation and detection of all targets tested. Today, these modules have been integrated in a beacon designed by CSTB (Fig. 5) and enable automation of all the steps leading to the calculation of the Fungal Contamination Index. 


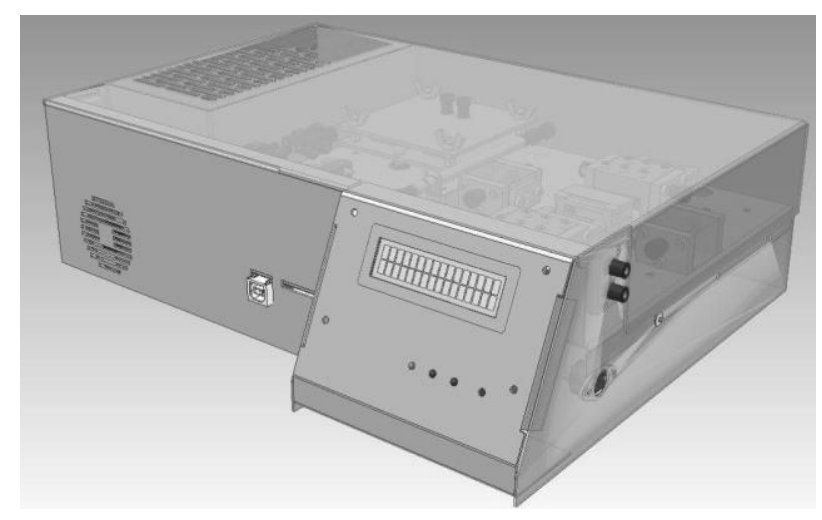

Fig. 5: 3D picture of indoor environments' fungal development monitoring device

This device for detecting fungal contamination was the subject of patents by the CSTB (Moularat, Joblin et al. 2010; Moularat, Joblin et al. 2011).

Research is in progress in order to develop new sensitive layers adapted to other gases and metrology dedicated to the particles exposure measurement.

\section{ACKNOWLEDGEMENTS}

We would like to thank:

- the Laboratoire de Physicochimie des Polymères et des Interfaces at the University of Cergy Pontoise (France) for their assistance for polymers used during this study. -the Laboratoire d'Electronique, Systèmes de COmmunication et Microsystèmes (ESYCOM), Champs-sur-Marne(France), for their help in microstructures fabrication. 


\section{REFERENCES}

Bailey, A., A. M. Pisanelli, et al. (2008). "Development of conducting polymer sensor arrays for wound monitoring." Sensors and Actuators B: Chemical 131(1): 5-9.

Canhoto, O., F. Pinzari, et al. (2004). "Application of electronic nose technology for the detection of fungal contamination in library paper." International Biodeterioration \& Biodegradation 54(4): 303-309.

Dutta, R., D. Morgan, et al. (2005). "Identification of Staphylococcus aureus infections in hospital environment: electronic nose based approach." Sensors and Actuators B: Chemical 109(2): 355-362.

Escamilla-Garcia, B. (1997). Étiologie comparative des maladies respiratoires d'une population d'enfants à Montréal et à Vancouver : regard sur les moisissures de l'air intérieur., Université de Montréal.

Garrett, M., P. Rayment, et al. (1998). "Indoor airborne fungal spores, house dampness and associations with environmental factors and respiratory health in children." Clin Exp Allergy 28: 459-467.

Gibson, T. D., O. Prosser, et al. (1997). "Detection and simultaneous identification of microorganisms from headspace samples using an electronic nose." Sensors and Actuators B: Chemical 44(1-3): 413-422.

Hamilton, S., M. J. Hepher, et al. (2006). "Detection of Serpula lacrymans infestation with a polypyrrole sensor array." Sensors and Actuators B: Chemical 113(2): 989-997.

Hulin, M., D. Caillaud, et al. (2010). "Indoor air pollution and childhood asthma: variations between urban and rural area " Indoor air 20: 502-514.

Hulin, M., S. Moularat, et al. (2013). "Positive associations between respiratory outcomes and fungal index in rural inhabitants of a representative sample of French dwellings." International Journal of Hygiene and Environmental Health 216: $155-162$.

Joblin, Y. (2011). Elaboration d'un microsystème d'analyse de l'air destiné à la détection rapide d'un développement fongique dans les espaces clos, Université Paris-Est Marne-la-Vallée / Ecole d'ingénieur ESIEE.

Joblin, Y., S. Moularat, et al. (2010). "Detection of moulds by volatile organic compounds: Application to heritage conservation." International Biodeterioration \& Biodegradation 64(3): 210-217.

Kilpelainen, M., E. Terho, et al. (2001). "Home dampness, current allergic diseases, and respiratory infections among young adults." Thorax 56: 462-467.

Korpi, A., J. Kasanen, et al. (1999). "Sensory irritating potency of some microbial volatile organic compound (MVOCs) and a mixture of five MVOCs." Archives of Environmental Health 54(5): 347-352.

Korpi, A., A. L. Pasanen, et al. (1997). "Microbial growth and metabolism in house dust." International Biodeterioration \& Biodegradation 40(1): 19-27.

Kuhn, I., V. Hana, et al. (1995). "Determination of ochratoxin A in bile of swine by high-performance liquid chromatography." Journal of chromatography B 668 : 333-337. 
Lebart, L., A. Morineau, et al. (1997). Statistique exploratoire multidimensionnelle $4^{\text {eme }}$ édition. Paris.

Li, B., S. Santhanam, et al. (2007). "Inkjet printed chemical sensor array based on polythiophene conductive polymers." Sensors and Actuators B: Chemical 123(2): 651-660.

Moularat, S. (2007). Procédé de détection d'une contamination fongique. INPI. France.

Moularat, S., M. Hulin, et al. (2011). "Airborne fungal volatile organic compounds in rural and urban dwellings: Detection of mould contamination in 94 homes determined by visual inspection and airborne fungal volatile organic compounds method." Science of The Total Environment 409: 2005-2009.

Moularat, S., Y. Joblin, et al. (2010). Dispositif de détection d'une contamination fongique. France. INPI.

Moularat, S., Y. Joblin, et al. (2011). Développement d'un microsystème de détection.

Moularat, S., E. Robine, et al. (2008). "Detection of fungal development in a closed environment through the identification of specific VOC: Demonstration of a specific VOC fingerprint for fungal development." Science of the Total Environment 407: 139-146.

Moularat, S., E. Robine, et al. (2008). "Detection of fungal development in closed spaces through the determination of specific chemical targets." Chemosphere 72(2): 224-232.

Nguyen, T.-P., S. Moularat, et al. (2012). DECAGRAPH, détection précoce des contaminants des collections graphiques. Colloque SMPC2, Sciences des matériaux du patrimoine culturel, Paris.

Nist'98 (1998). Mass spectral library (vers 1.7).

Pirhonen, I., A. Nevalainen, et al. (1996). "Home dampness, moulds and their influence on respiratory infections and symptoms in adults in Finland." European Respiratory Journal 9(12): 2618-2622.

Reidy, S., G. Lambertus, et al. (2006). "High-performance, static-coated silicon microfabricated columns for gas chromatography." Analytical chemistry 78(8): 2623-2630.

Serrano, G., S. M. Reidy, et al. (2009). "Assessing the reliability of wall-coated microfabricated gas chromatographic separation columns." Sensors and Actuators B: Chemical 141(1): 217-226.

Tsongas, G. (1994). Crawl space moisture conditions in new and existing Northwest homes. Recommended Practices for Controlling Moisture in Crawl Spaces. R. a. A.-C. E. American Society of Heating. Atlanta, Technical Data Bulletin. 10: 4148.

Wessen, B., G. Ström, et al. (1995). MVOC-profiles-a tool for indoor-air quality assessment. An Integrated Approach. L. Morawska. Amsterdam, Indoor Air Quality: 67-70.

Wilkins, K. (2002). Microbial VOC (MVOC) in buildings, their properties and potential use. 9th International Conference on Indoor Air Quality and Climate, Monterey, California.

Xiong, Z. and C. Liu (2012). "Optimization of inkjet printed PEDOT:PSS thin films through annealing processes." Organic Electronics 13(9): 1532-1540. 